\title{
P-0748 - First stratified diabetes prevalence data for Republic of Macedonia derived from the National eHealth System
}

Smokovski Ivica ${ }^{1,2}$, Milenkovic Tatjana ${ }^{1,3}$

${ }^{1}$ University Clinic of Endocrinology, Diabetes and Metabolic Disorders Skopje, 2 University Goce Delcev Stip,

3 University Sts Cyrillus and Methodius Skopje, Republic of Macedonia

Background: Republic of Macedonia is estimated to have the third highest diabetes prevalence in Europe [1]. National eHealth System has been introduced in the country since 2013 covering all citizens across primary, secondary and tertiary healthcare, integrating diabetes related data, and, for the first time, enabling stratified diabetes prevalence calculation based on diagnosed diabetes cases [2].

Aims: To find diabetes prevalence of diagnosed cases, stratified by gender, age and urban or rural population in Republic of Macedonia, based on data derived from the National eHealth System.

Method: National eHealth System was searched for all patients with ICD-10 diagnoses $\mathrm{E} 10-\mathrm{E} 14$ in their electronic healthcare records. Data records derived from the National eHealth System contained patient's gender, date of birth, place of living and ICD-10 code.

Results: Urban, rural and total population diagnosed with diabetes by age and gender are presented in Table 1,2 and $3 ; n$ is the number of cases with diabetes in the group, and $N$ is the total number of individuals (with and without diabetes) in the group. Total number of diagnosed diabetes cases was 84,568: 36,119 males (42.71\%) and 48,449 females $(57.29 \%)$. Diabetes prevalence of diagnosed cases in population 20-79 years was $5.01 \%(n=78,233 ; N=1,562,203)$.

\section{References:}

1. International Diabetes Federation. IDF Diabetes Atlas. 7th ed. Brussels, Belgium: International Diabetes Federation, 2015. http://www.idf.org/diabetesatlas (accessed April 26, 2017).

2. Smokovski I, Milenkovic T, Trapp C, Mitov A. Diabetes Care in Republic of Macedonia: Challenges and Opportunities. Annals of Global Health; 2015; 81(6): 792-802.
Table 1. Urban population diagnosed with diabetes by age and gender

\begin{tabular}{|l|c|c|c|c|c|c|c|c|c|}
\hline $\begin{array}{l}\text { Age Groups } \\
\text { (years) }\end{array}$ & & Male & & & Female & & & Total & \\
\hline & $n$ & $N$ & $\%$ & $n$ & $N$ & $\%$ & $n$ & $N$ & $\%$ \\
\hline$<20$ & 275 & 194,362 & $0.14 \%$ & 269 & 182,757 & $0.15 \%$ & 544 & 377,119 & $0.14 \%$ \\
\hline $20-39$ & 1,097 & 257,387 & $0.43 \%$ & 1,114 & 247,693 & $0.45 \%$ & 2,211 & 505,080 & $0.44 \%$ \\
\hline $40-59$ & 8,792 & 233,220 & $3.77 \%$ & 8,887 & 231,703 & $3.84 \%$ & 17,679 & 464,923 & $3.80 \%$ \\
\hline $60-79$ & 14,529 & 128,512 & $11.31 \%$ & 20,316 & 149,747 & $13.57 \%$ & 34,845 & 278,259 & $12.52 \%$ \\
\hline $80+$ & 1,655 & 14,178 & $11.67 \%$ & 2,652 & 21,970 & $12.07 \%$ & 4,307 & 36,148 & $11.91 \%$ \\
\hline Total & 26,348 & 827,659 & $3.18 \%$ & 33,238 & 833,870 & $3.99 \%$ & 59,586 & $1,661,529$ & $3.59 \%$ \\
\hline
\end{tabular}

Table 2. Rural population diagnosed with diabetes by age and gender

\begin{tabular}{|l|c|c|c|c|c|c|c|c|c|}
\hline $\begin{array}{l}\text { Age Groups } \\
\text { (years) }\end{array}$ & & Male & & & Female & & & Total & \\
\hline & $n$ & $N$ & $\%$ & $n$ & $N$ & $\%$ & $n$ & $N$ & $\%$ \\
\hline$<20$ & 2 & 63,309 & $0.00 \%$ & 3 & 58,423 & $0.01 \%$ & 5 & 121,732 & $0.00 \%$ \\
\hline $20-39$ & 430 & 75,572 & $0.57 \%$ & 561 & 68,563 & $0.82 \%$ & 991 & 144,135 & $0.69 \%$ \\
\hline $40-59$ & 3,888 & 59,532 & $6.53 \%$ & 4,994 & 55,842 & $8.94 \%$ & 8,882 & 115,374 & $7.70 \%$ \\
\hline $60-79$ & 4,989 & 25,677 & $19.43 \%$ & 8,636 & 28,755 & $30.03 \%$ & 13,625 & 54,432 & $25.03 \%$ \\
\hline $80+$ & 462 & 3,562 & $12.97 \%$ & 1,017 & 4,952 & $20.54 \%$ & 1,479 & 8,514 & $17.37 \%$ \\
\hline Total & 9,771 & 227,652 & $4.29 \%$ & 15,211 & 216,535 & $7.02 \%$ & 24,982 & 444,187 & $5.62 \%$ \\
\hline
\end{tabular}

Table 3. Total population diagnosed with diabates by age and gender

\begin{tabular}{|c|c|c|c|c|c|c|c|c|c|}
\hline $\begin{array}{l}\text { Age } \\
\text { Groups } \\
\text { (years) }\end{array}$ & & Male & & & Female & & & Total & \\
\hline & $n$ & $N$ & $\%$ & $n$ & $N$ & $\%$ & $n$ & $N$ & $\%$ \\
\hline$<20$ & 277 & 257,671 & $0.11 \%$ & 272 & 241,180 & $0.11 \%$ & 549 & 498,851 & $0.11 \%$ \\
\hline $20-39$ & 1,527 & 332,959 & $0.46 \%$ & 1,675 & 316,256 & $0.53 \%$ & 3,202 & 649,215 & $0.49 \%$ \\
\hline $40-59$ & 12,680 & 292,752 & $4.33 \%$ & 13,881 & 287,545 & $4.83 \%$ & 26,561 & 580,297 & $4.58 \%$ \\
\hline $60-79$ & 19,518 & 154,189 & $12.66 \%$ & 28,952 & 178,502 & $16.22 \%$ & 48,470 & 332,691 & $14.57 \%$ \\
\hline $80 \div$ & 2,117 & 17,740 & $11.93 \%$ & 3,669 & 26,922 & $13.63 \%$ & 5,786 & 44,662 & $12.96 \%$ \\
\hline Total & 36,119 & $1,055,311$ & $3.42 \%$ & 48,449 & $1,050,405$ & $4.61 \%$ & 84,568 & $2,105,716$ & $4.02 \%$ \\
\hline
\end{tabular}

Discussion: These were the first findings on diabetes prevalence of diagnosed cases in Republic of Macedonia derived from the National eHealth System, stratified by age, gender and urban or rural population. Diabetes prevalence of diagnosed cases in total population was higher in females than in males $(4.61 \%$ vs $3.42 \%)$; it was higher in rural than in urban population $(5.62 \%$ vs $3.59 \%$ ) and was highest in the age group 60-79 years $(14.57 \%)$. These data could enable more precise estimation of the total diabetes prevalence in the country including both diagnosed and undiagnosed cases, and further analysis of the risk factors leading to higher diabetes prevalence in females and rural population in Republic of Macedonia.
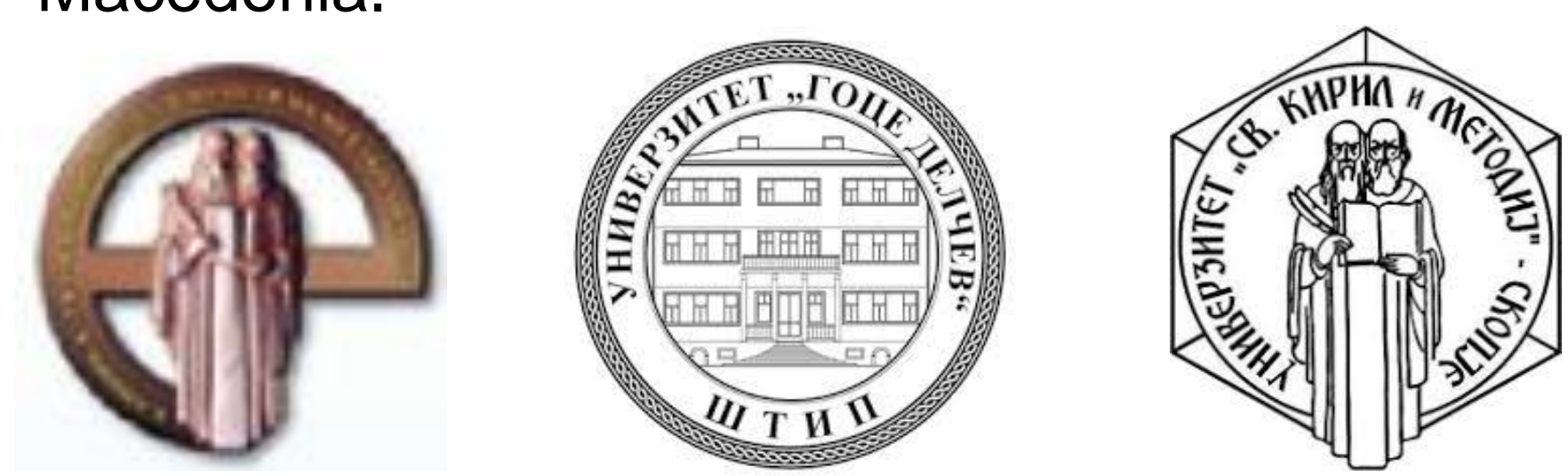